\title{
Analyzing the features of mughal garden design: case of Taj Mahal, Agra, India
}

\begin{abstract}
Islamic garden is a place for rest and reflection, and a reminder to paradise. The themes of an Islamic garden are shade and water element. The Islamic Gardens are influenced by the historic Islamic Garden that majority from Spain, Iran, and Mughal India. Those are among the recognized gardens design during Islamic Civilization and remain as a legacy of the influential era of Islamic rulers. However the gardens have different culture influences that make each of the gardens are different in their design. This paper is to analyzing the features used in the garden design of Mughal Garden: Taj Mahal, Agra, India. The study used a content analysis and observation of features used in the design for the garden. The outcome of this study will enhance the understanding and appreciation towards Islamic garden design as an Islamic cultural heritage for the next generation.
\end{abstract}

Keyword: Islamic garden; Mughal garden; Taj Mahal 